\title{
Clinical utility of pre-transplant ophthalmic consultation for lung transplant recipients: implications in the COVID-19 pandemic era
}

\author{
Tavish Nanda $^{1} \cdot$ Mark P. Breazzano $^{1,2} \cdot$ Srilaxmi Bearelly ${ }^{1}$
}

Received: 7 June 2020 / Revised: 7 June 2020 / Accepted: 9 June 2020 / Published online: 22 June 2020

(C) Springer-Verlag GmbH Germany, part of Springer Nature 2020

\section{Dear Editor,}

A screening examination is among the most common ophthalmologic inpatient consultations in the hospital system [1]. Its indiscriminate practice has persisted for decades, though little is supported by evidence-based epidemiologic or outcomes data. Ophthalmologic screening for Candida bloodstream infections, for example, is historically engrained in medicine checklists, largely influenced by the Infectious Disease Society of America [2]. When reviewed systemically, however, asymptomatic screening did not demonstrate an association with better ophthalmic outcomes [3]. Only now are institutions altering guidelines related to this topic.

In this SARS-Cov-2 (COVID-19) era, many reflexive ophthalmologic screening examinations are undergoing increased scrutiny, and though the topic is not unique to the current pandemic; this process has spurred the reexamination of "essential" vs. "non-essential" interventions [1]. This reappraisal is especially pertinent for inpatient lung transplant patients, many of whom are acutely ill and immunocompromised. Pathogen transmissibility across ophthalmic equipment, for example, has resulted in infection and mortality in a similarly vulnerable group [4]. Globally, institution-specific guidelines that include pre-transplant eye examination remain a prerequisite to transplantation, even though justification for this evaluation is questionable [5, 6].

As part of an institutional-review board approved effort to identify non-essential interventions, we reviewed

Tavish Nanda

tn2290@cumc.columbia.edu

1 Columbia University Irving Medical Center, New York-Presbyterian Hospital, Edward S. Harkness Eye Institute, 635 W 165th St, New York, NY 10032, USA

2 Department of Ophthalmology, New York University Langone Health, New York University School of Medicine, New York, NY, USA all lung transplant ophthalmologic consultations from 2014 to 2019 to determine their continued utility in the setting of the COVID-19 pandemic. In total, 154 consults were identified, 89 pre-transplant, and 65 post-transplant (Table 1). $94.8 \%$ of pre-transplant screening evaluations were asymptomatic on ocular review of systems. Average visual acuity was 20/32 \pm 46 and average intraocular pressure was $14.5 \pm 3.1 \mathrm{mmHg}$. No findings served as reasonable contraindications to acute lung transplantation (Table 2). Only 18/85 (21\%) of patients received a lung transplant (mean 33.8 days post-consultation [12-98]), 43/84 (51\%) were discharged after clinical improvement (mean 27.8 days postconsultation [1-243]), and 23/84 (27\%) patients died (mean 16.6 days post-consultation [0-79]). Eleven patients $(13.1 \%)$ were deemed ineligible due to poor health or psychosocial instability, after ophthalmologic evaluation. The remaining 65 consultations occurred on average 1.7 years ( 3 days -11 years) post-transplantation. $35 / 65(52.3 \%)$ were symptomatic, with the most common complaints including blurry vision (20\%), redness/eye pain $(17 \%)$, and reduced vision (8\%). Thirty-one consults were for asymptomatic screening examinations, such as cytomegalovirus bloodstream infection, of which $0 \%$ had relevant findings. 8/65 had non-benign ocular findings on examination; two vascular incidents, two with disc edema from diabetes and elevated intracranial pressure, three with medication-induced optic atrophy, and one with a visual field defect related to meningitis (Table 2). All 8 of these patients were acutely symptomatic.

Based on these data, the utility of an urgent pre-transplant examination in the inpatient setting appears impractical, as only $21 \%$ received a transplant during their hospitalization, and $0 \%$ had pertinent ophthalmic findings. There are also no set guidelines for routine ophthalmologic follow-up posttransplantation, which is problematic when recommending a pre-transplant evaluation, as any non-urgent pertinent positives (including cataract or steroid-induced glaucoma) may 
Table 1 Baseline patient demographics by consult (154 total)

\begin{tabular}{|c|c|}
\hline & \# of Consults \\
\hline Gender & $\begin{array}{l}\text { Male: } 80 \\
\text { Female: } 74\end{array}$ \\
\hline Age (years) & Avg. $50.6(15-75)$ \\
\hline Ethnicity & $\begin{array}{l}\text { Caucasian: } 96 \\
\text { Hispanic: } 30 \\
\text { African American: } 5 \\
\text { Asian: } 3 \\
\text { Unknown: } 20\end{array}$ \\
\hline Diagnosis (lung) & $\begin{array}{l}\text { Cystic fibrosis: } 33 \\
\text { Chronic obstructive pulmonary } \\
\quad \text { disease/emphysema: } 14 \\
\text { Interstitial lung disease: } 42 \\
\text { Pulmonary arterial hypertension: } 10 \\
\text { Idiopathic pulmonary fibrosis: } 17 \\
\text { Scleroderma/connective tissue disease: } 8 \\
\text { Sjogren's syndrome: } 4 \\
\text { Systemic lupus erythematosus: } 2 \\
\text { Primary ciliary dyskinesia: } 1 \\
\text { Usual interstitial pneumonia: } 3 \\
\text { Pulmonary embolus: } 1 \\
\text { Non-specific interstitial pneumonia: } 4 \\
\text { Idiopathic pleuroparenchymal fibroelastosis: } 1 \\
\text { Hypersensitivity pneumonitis: } 1 \\
\text { Hermansky-Pudlak: } 2 \\
\text { Other: } 8\end{array}$ \\
\hline Admission & $\begin{array}{l}\text { Transplant rejection: } 6 \\
\text { Acute on chronic respiratory failure: } 65 \\
\text { Post-transplant care: } 16 \\
\text { CMV viremia: } 9 \\
\text { Other infection: } 18 \\
\text { Cardiogenic: } 9 \\
\text { Altered mental status: } 4 \\
\text { Other: } 27\end{array}$ \\
\hline
\end{tabular}

not receive appropriate follow-up [7]. Additionally, none of the presumed "absolute contraindications" to transplantation, such as incidental ocular malignancy or asymptomatic intraocular infection, meet epidemiological screening criteria [3, 8]. Our data are similar to published numbers by Sere et al., who also found no ocular contraindications to lung transplantation $(0 / 295)$, resulting in a systematic change to the national Dutch lung transplant program that no longer requires inpatient ophthalmologic screening [9]. Therefore, we support a symptom-based approach to both pre- and post-transplant consultation in order to minimize harm through unnecessary exposures and potential medication side effects (e.g., phenylephrine-induced reflex bradycardia) [4, 10]. As COVID-19 reshapes how physicians and institutions address
Table 2 Pre- and post-transplant ophthalmic findings

\begin{tabular}{lrr}
\hline Ocular findings & Pre & Post \\
\hline None & 41 & 26 \\
Nuclear sclerosis & 8 & 5 \\
Posterior subcapsular cataract (PSC) & 2 & 1 \\
Trace nuclear sclerosis & 9 & 4 \\
Posterior vitreal detachment & 0 & 3 \\
Exposure keratopathy & 0 & 4 \\
Dry eye syndrome & 3 & 3 \\
Lattice degeneration & 2 & 0 \\
Vascular changes & 5 & 4 \\
Peripheral drusen & 3 & 2 \\
Retinal nevi & 2 & 0 \\
Ocular albinism & 2 & 0 \\
Retinal vascular incident & 0 & 2 \\
Optic neuropathy & 0 & 3 \\
Disc edema & 0 & 2 \\
Visual field defect & 0 & 1 \\
Other & 16 & 9 \\
\hline
\end{tabular}

certain care models, we expect additional, similar interventions to be re-evaluated in a data-driven fashion.

Funding information The New York Community Trust-Frederick $\mathrm{J}$ and Theresa Dow Wallace Fund, Columbia University (S.B.).

Availability of data and material Not Applicable.

\section{Compliance with ethical standards}

Conflicts of interest/competing interests The authors declare that they have no conflicts of interest.

Ethics approval This was a Columbia University Irving Medical Center institutional review board approved protocol \#AAAS6456.

Code availability Not Applicable.

Study institution Columbia University Irving Medical Center, New York, NY, USA.

\section{References}

1. Oh DJ, Kanu LN, Chen JL, Aref AA, Mieler WF, MacIntosh PW (2019) Inpatient and emergency room ophthalmology consultations at a tertiary care center. J Ophthalmol. https://doi.org/10.1155/ 2019/7807391

2. Pappas PG, Kauffman CA, Andes DR, Clancy CJ, Marr KA, Ostrosky-Zeichner L, Reboli AC, Schuster MG, Vazquez JA, Walsh TJ, Zaoutis TE (2016) Clinical practice guideline for the management of candidiasis: 2016 update by the Infectious Diseases Society of America. Clin Infect Dis 62(4):e1-e50 
3. Breazzano MP, Day HR, Bloch KC, Tanaka S, Cherney EF, Sternberg P, Donahue SP, Bond JB (2019) Utility of ophthalmologic screening for patients with Candida bloodstream infections: a systematic review. JAMA Ophthalmol 137(6):698-710

4. Sammons JS, Graf EH, Townsend S, Hoegg CL, Smathers SA, Coffin SE, Williams K, Mitchell SL, Nawab U, Munson D, Quinn G (2019) Outbreak of adenovirus in a neonatal intensive care unit: critical importance of equipment cleaning during inpatient ophthalmologic examinations. Ophthalmology 126(1):137-143

5. American Society of Transplantation (2006) Getting a new lung. Facts about lung transplants. American Society of Transplantation. https://www.myast.org/sites/default/files/pdfs/getting_new_lung $0 . p d f$

6. Hirche TO, Knoop C, Hebestreit H, Shimmin D, Solé A, Elborn JS, Ellemunter H, Aurora P, Hogardt M, Wagner TO, Ecorn-Cf study group $(2014,2014)$ Practical guidelines: lung transplantation in patients with cystic fibrosis. Pulm Med. https://doi.org/10.1155/ 2014/621342

7. Skalka HW, Prchal JT (1980) Effect of corticosteroids on cataract formation. AMA Arch Ophthalmol 98(10):1773-1777

8. Egan KM, Seddon JM, Glynn RJ, Gragoudas ES, Albert DM (1988) Epidemiologic aspects of uveal melanoma. Surv Ophthalmol 32(4):239-251

9. Seré M, Ouwens JP, van der Bij W, Hardus PL (2004) The value of routine ophthalmological tests in the screening for lung transplantation. Transpl Int 17(12):859-861

10. Kumar V, Schoenwald RD, Chien DS, Packer AJ, Choi WW (1985) Systemic absorption and cardiovascular effects of phenylephrine eyedrops. Am J Ophthalmol 99(2):180-184

Publisher's note Springer Nature remains neutral with regard to jurisdictional claims in published maps and institutional affiliations. 